\title{
Modeling inside building electromagnetic wave propagation using a finite difference time domain method
}

\author{
J. Sosa-Pedroza, F. Martínez-Zúñiga \& S. Coss-Domínguez \\ Departamento de Telecomunicaciones ESIME IPN, Mexico
}

\begin{abstract}
Finite Difference Time Domain Method (FDTDM) is a powerful tool to model electromagnetic propagation; once the medium has been defined by its conductivity $(\sigma)$, permittivity $(\varepsilon)$ and permeability $(\mu)$, for each position in the computational volume, is possible to "see" the propagation electromagnetic behavior. We have used FDTDM to model waveguides, slot antennas, field effects over different materials and even propagation effects in nanometric spaces. As obstacles produce reflection, refraction and diffraction phenomena, modifying scattering field in free space zones, knowledge of propagation behavior is very useful to define antenna positions and other system characteristics, for WiFi, cellular telephony and other mobile communication systems. We present in this paper, results of EM propagation modeling inside a building, where furniture, structure, walls and any other obstacle has been defined by their EM constants: $\sigma, \varepsilon, \mu$. Modeling shows how EM field is distributed all over the chosen zone, giving an almost exact quantification of field magnitude in each point. Modeling uses Yee algorithm to transform Differential Maxwell Equations into Finite Difference Equations, capable to be handled by the computer program developed by us. The source we used was a $2.4 \mathrm{GHz} \mathrm{WiFi}$ access point, allowing us to compare computational results with direct measurements in the experimental area. Each computational point was a $0.6 \mathrm{~cm}(\lambda / 20)$ per side cube, over a zone of $9 \times 4 \times 3 \mathrm{~m}$. Results are presented in both, as images showing field distribution in representative areas of analysis zone, and linear graphics comparing acquired computational and measurement data, showing a no more than $3 \mathrm{~dB}$ difference between them.
\end{abstract}

Keywords: finite difference time domain method, propagation in building areas, electromagnetic field propagation. 


\section{Introduction}

As is known the Finite Difference Time Domain Method (FDTDM) transforms Maxwell differential equations in finite difference equations to be modelled using computational methods [1], in this way is possible to analyse any electromagnetic device or propagation phenomena.

We have been working with FDTDM, analysing many kind of electromagnetic devices and propagation phenomena [2-4]. We present in this paper analysis of electromagnetic propagation in building areas, substituting electromagnetic characteristics $(\varepsilon, \mu, \sigma)$ of furniture, walls, metals, windows etc. Results show how obstacles affect electromagnetic wave; as we have point calculation in the computational volume, is possible to predict field in any desired place.

The experiment is developed in a three rooms of $3.5 \mathrm{~m}$ tall with a total area of $9 \times 4 \mathrm{~m}$. Simulation is made over cubes of $0.6 \mathrm{~cm}$ by side $(\Delta=\lambda / 20)$, measurement comparing test was made over different height layers, to test simulation experiment.

To test predicted results we compare them with measurements around the experiment zone. The chosen source is a $\mathrm{WiFi}$ access point working at $2.4 \mathrm{GHz}$, to make easy the measurement test and comparison with simulation process.

\section{The finite difference time domain method}

FDTD was presented in 1966 by Yee [5]. The method models space derivatives of Maxwell equations as a 3D central finite difference approximation in a regular Cartesian mesh. The time derivatives are implemented in a leap-frog scheme, i.e. the electric field is obtained in a time slot and then the magnetic field in the next, considering all boundary conditions in the computational space.

Fundamentals of Yee algorithm are:

- $\quad$ Electric and magnetic fields are modelled, in time and space, using Maxwell equations.

- $\quad \mathbf{E}$ and $\mathbf{H}$ components are centered in a three dimensional space i.e. each $\mathbf{E}$ component is surrounded by four sides of two $\mathbf{H}$ components, in the same way each $\mathbf{H}$ component is surrounded by four sides of two $\mathbf{E}$ components.

- Time components are also centered in a leap-frog scheme.

- $\quad$ The $\mathbf{E}$ data in the computational space is stored for each time slot using the previously stored data of $\mathbf{H}$, in the same way all $\mathbf{H}$ data is obtained and stored using the $\mathbf{E}$ data, in an iterative procedure.

Let a space region, free of electric or magnetic sources, made of any kind of material: conductors, dielectrics, ferromagnetic, etc.; Maxwell equations describes propagation of electromagnetic field in that media:

$$
\begin{gathered}
\frac{\partial \bar{B}}{\partial t}=-\nabla \times \bar{E}-\bar{J}_{m} \\
\frac{\partial \bar{D}}{a}=\nabla \times \bar{H}-\bar{J}_{e}
\end{gathered}
$$

Equations (1) and (2) are expanded in a three dimensional space $(x, y, z)$ (we show only two components) as: 


$$
\begin{gathered}
\frac{\partial H_{x}}{\partial}=\frac{1}{\mu}\left(\frac{\partial E_{y}}{\partial z}-\frac{\partial E_{z}}{\partial y}-\rho^{\prime} H_{x}\right) \\
\frac{\partial E_{x}}{\partial t}=\frac{1}{\varepsilon}\left(\frac{\partial H_{z}}{\partial y}-\frac{\partial H_{y}}{\partial z}-\sigma E_{x}\right)
\end{gathered}
$$

$\varepsilon$ is the dielectric permittivity, $\mu$ the magnetic permeability, $\sigma$ electric conductivity and $\rho$ ' the magnetic resistivity. According with Yee notation, any point with arbitrary electric and magnetic characteristics in the uniform space mesh, made by cells as the one shown in Figure 1, are defined as:

$$
(i, j, k)=(i \Delta x, j \Delta y, k \Delta z)
$$

and any function $F$ in a discrete point in time and space in the mesh as:

$$
F_{i, j, k}^{n}=F(i \Delta x, j \Delta y, k \Delta z, n \Delta t)
$$

$\Delta \mathrm{x}, \Delta \mathrm{y}, \Delta \mathrm{z}$ and $\Delta \mathrm{t}$ are incremental components in space and time, with $i, j, k$ and $n$ as whole numbers.

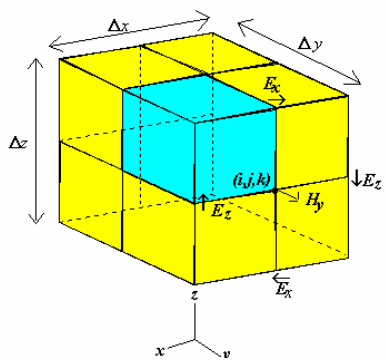

Figure 1: Yee cell.

Considering all definitions, expressions of central finite difference for space and time derivatives of $\mathrm{F}$ in $\mathrm{x}$ direction is:

$$
\frac{\partial F(i \Delta x, j \Delta y, k \Delta z, n \Delta t)}{\partial x}=\frac{F_{i+1 / 2, j, k}^{n}-F_{i-1 / 2}^{n}, j, k}{\Delta x}+O\left[\left(\Delta x^{2}\right)\right]
$$

In the same way the time derivative of $\mathrm{F}$, for the $(\mathrm{i}, \mathrm{j}, \mathrm{k})$ point is:

$$
\frac{\partial F(i \Delta x, j \Delta y, k \Delta z, n \Delta t)}{2}=\frac{F_{i, j, k}^{n+1 / 2}-F_{i, j, k}^{n-1 / 2}}{\Delta t}+O\left[\left(\Delta t^{2}\right)\right]
$$

Equations (7) and (8) are obtained with expansion of derivatives in a Taylor series, $\mathrm{O}$ representing the residual of high order terms. Using these equations in the Maxwell differential equations (3) and (4) and with a simple algebraic work, the algorithm for $\mathbf{E}$ and $\mathbf{H}$ can be managed by the computer [6]. We present only two components, but all others have the same form:

$$
\begin{aligned}
& E_{x(i, j, k)}^{n+1}=\left(\frac{1-\frac{\sigma_{(i, j, k)} \Delta t}{2 \varepsilon_{(i, j, k)}}}{1+\frac{\sigma_{(i, j, k)} \Delta t}{2 \varepsilon_{(i, j, k)}}}\right) E_{x(i, j, k)}^{n} \\
& +\left(\frac{\frac{\Delta t}{\varepsilon_{(i, j, k)}}}{1+\frac{\sigma_{(i, j, k)} \Delta t}{2 \varepsilon_{(i, j, k)}}}\right) \cdot\left(\begin{array}{l}
\frac{H_{z(i, j+1 / 2, k)}^{n+1 / 2}-H_{z(i, j-1 / 2, k)}^{n+1 / 2}}{\Delta y} \\
-\frac{H_{y(i, j, k+1 / 2)}^{n+1 / 2}-H_{y(i, j, k-1 / 2)}^{n+1 / 2}}{\Delta z}
\end{array}\right)
\end{aligned}
$$




$$
\begin{aligned}
& H_{x(i, j, k)}^{n+1 / 2}=\left(\frac{1-\frac{\rho_{(i, j, k)}^{\prime} \Delta t}{2 \mu_{(i, j, k)}}}{1+\frac{\rho_{(i, j, k)}^{\prime} \Delta t}{2 \mu_{(i, j, k)}}}\right) H_{x(i, j, k)}^{n-1 / 2} \\
& +\left(\frac{\frac{\Delta t}{\mu_{(i, j, k)}}}{1+\frac{\rho_{(i, j, k)}^{\prime} \Delta t}{2 \mu_{(i, j, k)}}}\right) \cdot\left(\begin{array}{l}
\frac{E_{y(i, j, k+1 / 2)}^{n}-E_{y(i, j, k-1 / 2)}^{n}}{\Delta z} \\
-\frac{E_{z(i, j+1 / 2, k)}^{n}-E_{z(i, j-1 / 2, k)}^{n}}{\Delta y}
\end{array}\right)
\end{aligned}
$$

With equations (9) and (10) a new field component, in any point, depends only of its former value and the former values of the other field components in adjacent points; the finite difference equations represent the basic calculation in the mesh but is required other elements for modeling the field: a) Dimension of mesh elements, b) The initial source of field, and c) The boundary of computational space.

The mesh dimensions should avoid a significant field change, neither in a space or time interval, for a maximum c speed of electromagnetic field in the mesh, the stability criterion is:

$$
\Delta t \leq \frac{1}{c \sqrt{\frac{1}{(\Delta x)^{2}}+\frac{1}{(\Delta y)^{2}}+\frac{1}{(\Delta z)^{2}}}}
$$

The initial source field should accomplish two conditions: reduce the use of memory and time in the computational space and represent, as best as possible, the real source. We use in this work a small antenna (WiFi antenna), considered as a point source with a sinusoidal time variation:

$$
\left.E_{x}\right|_{i_{s}} ^{n}=E_{0} \operatorname{sen}\left(2 \pi f_{0} n \Delta t\right)
$$

For a good analysis, the computational domain should have enough size to contain the experiment structure, and the exterior perimeter must establish an adequate boundary to simulate an infinite extension of computational space. The boundary of computational space is limited by the absorption boundary condition $(\mathrm{ABC})$, avoiding field distortions due reflections at the end of computational mesh. $\mathrm{ABC}$ are differential operators canceling the wave at the end of computational volume. The $\mathrm{ABC}$ used in this experiment was the Berenger's PML (Perfect Matching Layer) [7].

Even we present only one component for each field in equations (9) and (10), the analysis is made in a 3D mesh, having three components for $\mathbf{E}$ and three for $\mathbf{H}$; each point in the computational space $(\mathrm{i}, \mathrm{j}, \mathrm{k})$ is defined by position and size given by equation (11), looking for a good approximation we define $\Delta=\lambda / 20$, which means a greater computational resources comparing with the usual $\Delta=\lambda / 10$ found in the literature.

We notice that each field point is defined by its electromagnetic characteristics $(\varepsilon, \mu, \sigma)$, knowing them is possible to substitute each one in equations (9) and (10) (also in the other four components), solving the Yee algorithm we obtain field for each point in the mesh; this field describes behavior of electromagnetic propagation all over the computational volume. 


\section{The experiment}

Our work was divided in two steps: the first one was simulation of area under test using FDTDM; second one was the measurement around the site, at different floor levels to compare with simulation results.

Figure 2 depicts the area under test. It is a three academic offices divided by wood, aluminium and glass walls, containing drawers, book shelves, work tables, computers and structural iron columns. The red point at the centre office depicts the source of electromagnetic field, it is a WiFi access point, Apple "airport extreme", working at $2.4 \mathrm{GHz}$, with a nominal output power of $20 \mathrm{dBmW}$, we used channel 8 at $2.447 \mathrm{GHz}$ with lower spurious interference signals.



Figure 2: The 3D site under test.

We developed the FDTD SW, to solve Maxwell Equations on a 3D scheme, each obstacle was located in the volume under test; site was divided geometrically in $0.6 \mathrm{~cm}$ per side cubes $(\lambda / 20)$, each one defined by their electromagnetic characteristics $(\varepsilon, \mu, \sigma)$. Figure 2 shows the site under analysis, with distribution of furniture and all other elements.

The 3D solution for a volume as big as the one analysed, requires a great amount of computational resources, to solve this problem we use OPEN MP parallel technique, and several hours, to obtain the desired results, considering up to $64 \mathrm{~Gb} / \mathrm{s}$ of RAM memory. Once the field for each point simulation was obtained, we convert data in images using Matlab; as an easy way to manage the huge quantity of data, we divide the area of analysis in 36 sections, as shown in Figure 3. Results are presented for magnetic field in three different heights, under, up and in the source levels. 


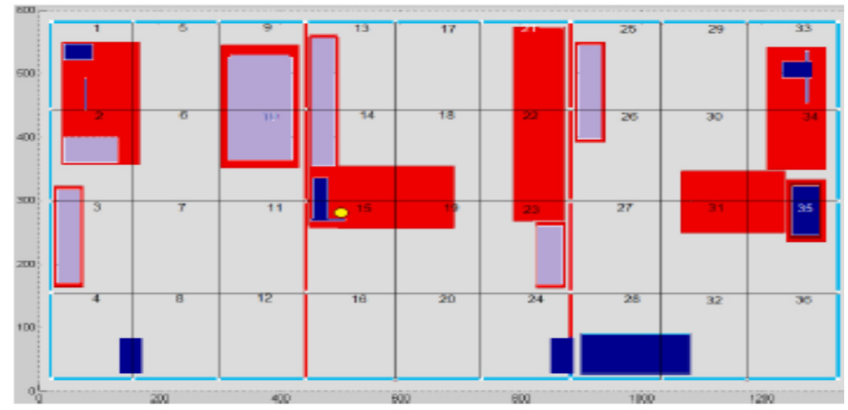

Figure 3: Area under test at source level.

\section{Simulation results}

Simulation results are presented in three height levels. Figure 4(a) shows results in sections 14 and 15 in the source plane. It is easy to see the effect of field reflection over the computer (metal) in front of source, defined by high power red lines, is also possible to see propagation over a book shelf with wave moving around the books; but the best and more useful view is the wave over the obstacle free space, where the field is really used; we observe an almost uniform wave propagation. Figure 4(b) describes propagation in sections 25 and 26, but $80 \mathrm{~cm}$ over the source level. We can see refraction and reflection in the glass window and propagation in other book shelf. We can see how the EM field is distorted by reflections in furniture, walls and iron columns.

Figure 4(c) shows field effects over section 1 displayed down the source level. Beneath the work table there were a metal equipment, showing an image with total reflection field. The second image in Figure 4(c), shows propagation over the work table; but again the most important view is propagation in the non-obstacle space.



(a)

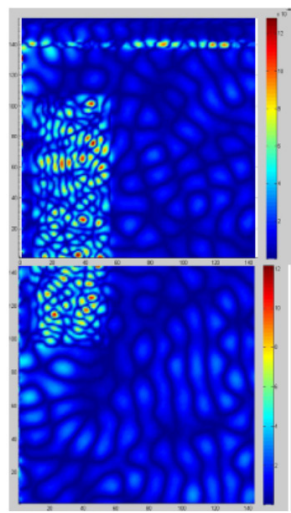

(b)

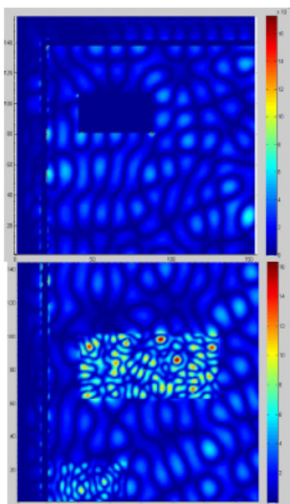

(c)

Figure 4: Matlab diagrams of field propagation over the analysis place. 


\section{Field measurement}

To test FDTDM we took measurements all over the site. To do so, we use an Anritsu Spectrum Master MS2723b (BTS) with an omnidirectional 2 dBi antenna. We use the same scheme of Figure 3, and results are presented in Table 1 for source level; Table 2 shows results $80 \mathrm{~cm}$ over the source level; and Table 3, 70 $\mathrm{cm}$ under source level. Tables are arranged using the presentation of Figure 3 for each section, i.e. column 1, row 1 correspond to section one, column 1 row 4 correspond to section 4 , column 9 row 1 defines section 33 and column 9 row 4 is for section 36 , and so on.

Table 1: Power measurement $(\mathrm{dBmW})$ at source level.

\begin{tabular}{|c|c|c|c|c|c|c|c|c|c|}
\hline & 1 & 2 & 3 & 4 & 5 & 6 & 7 & 8 & 9 \\
\hline 1 & -49.8 & -50.8 & -49.3 & -44.9 & -42.3 & -43.9 & -56.6 & -56.9 & -51.5 \\
\hline 2 & -49.8 & -50.8 & -46.2 & -36.2 & -38.6 & -39.8 & -48.9 & -51.0 & -50.8 \\
\hline 3 & -48.4 & -46.3 & -39.7 & -31.7 & -37.2 & -40.7 & -50.3 & -51.4 & -54.1 \\
\hline 4 & -48.2 & -46.9 & -46.8 & -41.4 & -43.6 & -43.2 & -50 & -53.8 & -52.4 \\
\hline
\end{tabular}

Table 2: $\quad$ Power measurement $(\mathrm{dBmW})$ over source level.

\begin{tabular}{|c|c|c|c|c|c|c|c|c|c|}
\hline & 1 & 2 & 3 & 4 & 5 & 6 & 7 & 8 & 9 \\
\hline 1 & -49.0 & -47.1 & -52.6 & -47.0 & -42.8 & -47.8 & -53.8 & -50.5 & -53.2 \\
\hline 2 & -52.0 & -47.9 & -51.7 & -48.4 & -40.8 & -42.1 & -49.6 & -50.6 & -55.9 \\
\hline 3 & -50.6 & -47.8 & -42.3 & -40.7 & -46.4 & -42.2 & -51.3 & -53.7 & -52.1 \\
\hline 4 & -53.3 & -46.9 & -46.7 & -43.5 & -46.2 & -43.3 & -51.9 & -54.2 & -52.0 \\
\hline
\end{tabular}

Table 3: Power measurement $(\mathrm{dBmW})$ below source level.

\begin{tabular}{|c|c|c|c|c|c|c|c|c|c|}
\hline & 1 & 2 & 3 & 4 & 5 & 6 & 7 & 8 & 9 \\
\hline 1 & -51.4 & -49.9 & -45.4 & -39.7 & -41.9 & -42.7 & -53.2 & -48.7 & -50.4 \\
\hline 2 & -52.9 & -50.5 & -47.7 & -35.5 & -38.2 & -42.7 & -46.0 & -44.1 & -52.2 \\
\hline 3 & -54.9 & -51.2 & -43.8 & -32.7 & -41.6 & -42.4 & -52.7 & -49.3 & -54.1 \\
\hline 4 & -51.2 & -53.9 & -50.6 & -39.0 & -43.5 & -44.8 & -47.6 & -52.9 & -57.7 \\
\hline
\end{tabular}

\section{Simulation and measurement comparison}

To compare simulation and measurement, we converted measured power into magnetic field, because FDTD results are for field and BTS measures power; results are shown in Figures 5-7. As seen the difference between FDTDM and 
measurement are very similar with only few $\mathrm{A} / \mathrm{m}$ for Magnetic field, mainly in the graphics at source level and under source level. The higher difference is in section 15 (source section) and both are almost the same in sections away from it (sections 35 and 36).

On the other hand, over the source level, differences are higher, as seen in Figure 7, although field behavior is very similar along the area of analysis. Is easy to obtain maximum difference into $\mathrm{dB}$, giving a value of $3 \mathrm{~dB}$.



Figure 5: Comparison of FDTD and measurement at source level.



Figure 6: Comparison of FDTD and measurement below source level. 


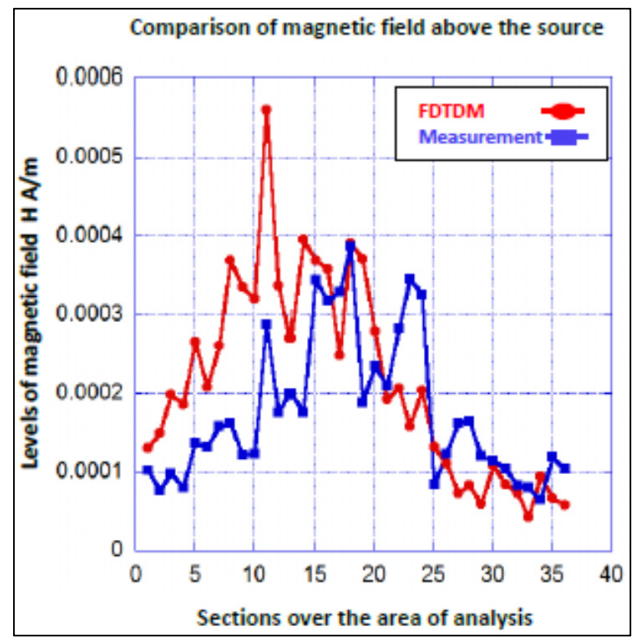

Figure 7: Comparison of FDTD and measurement over source level.

\section{Conclusion}

We have presented simulation over a building area using FDTDM, substituting characteristics of obstacles by their electromagnetic characteristics. To validate simulation we measure field over the area of analysis at different heights, concluding that both simulation and measurement are similar.

\section{References}

[1] A. Taflove, "Computational Electrodynamics: The Finite Difference Time Domain Method", Artech House, 2000, pp. 51-80.

[2] J. Sosa-Pedroza, M. Enciso-Aguilar, M. Benavides-Cruz, "Analysis of Terahertz Structures using FDTD Method”. PIERS 2013, Amsterdam Holland, August 2013.

[3] J. Sosa-Pedroza, S. Coss-Domínguez, "Efecto de propagación electromagnética usando el MDFDT con obstáculos de diferentes materiales", presentado en CNIES 2009, México, D.F.

[4] J. Sosa-Pedroza, M. Enciso-Aguilar, M. Benavides Cruz "A Parametric Analysis of Perfect Matched Layer Model of Finite Difference Time Domain Method", PIERS 2009, Moscow Russia 18-21 August 2009.

[5] K. S. Yee, "Numerical solution of initial boundary value problem involving Maxwell's equations in isotropic media" IEEE Transactions on antennas and Propagation, vol. 14, pp. 302-307, May 1966.

[6] D.P. Berenger, "A Perfectly Matched Layer for the Absorption of Electromagnetic Waves", Journal of Computational Physics. vol 114, Oct. 1994 pp. 185-200. 